
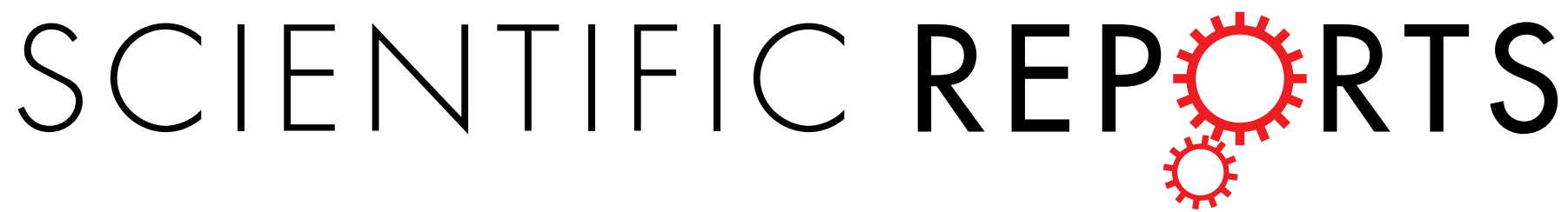

\title{
OPEN Immune Responses Induced by Recombinant Bacillus Subtilis Expressing the Hemagglutinin Protein of $\mathrm{H} 5 \mathrm{~N} 1$ in chickens
}

Accepted: 17 October 2016

Published: 16 December 2016

\section{Chunxiao Mou, Liqi Zhu, Jingjing Yang, Wenwen Xu, Xiaoying Cheng \& Oian Yang}

To develop an effective, safe, and convenient vaccine for the prevention of highly pathogenic avian influenza (HPAI) H5N1, we have constructed a recombinant Bacillus subtilis strain (B.S.-HA) expressing the hemagglutinin (HA) protein. Then we evaluated the immune function in chicken bone marrow derived dendritic cells (BM-DCs), and the immune response after oral immunization. Our results show that the recombinant Bacillus subtilis B.S.-HA could be sampled by BM-DCs in vitro and increase the BM-DCs major histocompatibility complex (MHC) II phenotype. The weight, height of the small intestine villus, and lymphoid tissue area of the ileum increased significantly in B.S.-HA immunized chickens $(P<0.05$ or $P<0.01)$. B.S.-HA induced the secretion of cytokines and the expression of Tolllike receptors in the trachea and small intestine $(P<0.05$ or $P<0.01)$. In addition, B.S.-HA elevated the specific IgA titers in the trachea, IgG and $\mathrm{HI}$ antibody titers in serum $(P<0.05$ or $P<0.01)$. Therefore, B.S.-HA provides a potential novel strategy and approach for developing an $\mathrm{H} 5 \mathrm{~N} 1$ vaccine.

The highly pathogenic avian influenza (HPAI) virus, H5N1, is a worldwide threat to the poultry industry. The virus evolved rapidly and shows high genetic diversity, a broad host range, and ongoing circulation in birds. $\mathrm{H} 5 \mathrm{~N} 1$ also has the potential to cause a human pandemic ${ }^{1}$. The current vaccination (intramuscular immunization) against HPAI is associated with reduced morbidity and mortality in poultry. However, intramuscular injection can inhibit growth and fail to induce sufficient mucosal immunity ${ }^{2}$. Many investigators are pursuing more convenient and economical routes to construct new vaccines, such as recombinant subunit vaccines using baculovirus $^{3}$, plasmid DNA ${ }^{4}$, or replication-incompetent adenoviral vectors ${ }^{5}$. H5N1 infects animals mainly through their respiratory and intestinal tracts ${ }^{6}$. Protective $\mathrm{H} 5 \mathrm{~N} 1$ antigens may induce an effective mucosal immune response to prevent invasion by $\mathrm{H} 5 \mathrm{~N} 1$, as many mucosa-associated lymphoid tissues underneath the epithelia of the respiratory and intestinal tracts. Therefore, developing a mucosal vaccine is a feasible strategy.

Bacillus subtilis is a nonpathogenic Gram-positive bacterium, a novel probiotic and biocontrol bacterium. B. subtilis offers unique resistance properties and survives under extreme conditions, such as extreme temperatures, desiccation, and exposure to noxious chemicals ${ }^{7}$. B. subtilis is widely used as a vehicle for heterologous antigen expression and protective immunization ${ }^{8,9}$. In addition, our previous study demonstrated that $B$. subtilis remarkably improved the immunoprotective efficacy of whole inactivated $\mathrm{H} 5 \mathrm{~N} 1$ influenza virus via oral immunization in chickens by enhancing local and systemic immune responses ${ }^{10}$. B. subtilis can also induce non-specific immune response against infection, increase IgA production, and regulate the balance of the Th1 and Th2 pathways ${ }^{11}$.

In this study, a recombinant B. subtilis strain (B.S.-HA) expressing hemagglutinin (HA) protein was constructed, and the efficacy of oral immunization of B.S.-HA was evaluated in chickens. B.S.-HA promoted growth, and induced cytokine secretion and expression of Toll-like receptors (TLRs). In addition, B.S.-HA could be sampled by chicken bone marrow-derived dendritic cells (BM-DCs), and it induced the expression of major histocompatibility complex (MHC) II.

\section{Materials and Methods}

Bacterial strains, plasmids, virus and animals. B. subtilis WB800N, and pHT43 plasmid were kindly provided by Dr. Xuewen Gao. pMD-19T-H5N1 plasmid was kindly provided by the Jiangsu Academy 


\begin{tabular}{|l|l|}
\hline Name & Sequence $\left(\mathbf{5}^{\prime} \mathbf{-}^{\prime} \mathbf{}\right)$ \\
\hline F1 & CGGATCCACCATGGAAACAACTCG \\
\hline R1 & GCCCGGGTTAAATGCAAATTCTGC \\
\hline TLR2 F & GATTGTGGACAACATCATTGACTC \\
\hline TLR2 R & AACGCTGCTTTCAAGTTTTCCC \\
\hline TLR4 F & TGACCTACCCATCGGACACT \\
\hline TLR4 R & CTCAGGGCATCAAGGTCTCC \\
\hline$\beta$-actin F & ATGAAGCCCAGAGCAAAAGA \\
\hline$\beta$-actin R & GGGGTGTTGAAGGTCTCAAA \\
\hline
\end{tabular}

Table 1. Oligonucleotides list.

of Agricultural Sciences. The inactivated avian influenza virus (IAIV) H5N1 was kindly provided by Qingdao Municipal Center for Disease Control \& Prevention. Specific-pathogen-free (SPF) chickens (Hyline) were kindly provided by Jiangsu Academy of Agricultural Sciences (Nanjing, China). The animal studies were approved by the Institutional Animal Care and Use Committee of Nanjing Agricultural University and followed the National Institutes of Health's guidelines for the performance of animal experiments.

Construction of recombinant $\boldsymbol{B}$. subtilis strains. To obtain recombinant $B$. subtilis $\mathrm{WB} 800 \mathrm{~N}$, a recombinant plasmid was constructed. Firstly, the HA fragments were amplified with primers F1/R1 (Table. 1) from pMD-19T-H5N1 plasmid, $p H T 43$ plasmid was digested by restriction enzyme BamHI and SmaI. Secondly, the fragment was purified and inserted into vector $p H T 43$ plasmid by T4 DNA ligase (Thermo Scientific) to generate the vector $p H T 43-H A$. The plasmid $p H T 43-H A$ was confirmed by restriction enzyme digestion with BamHI and SmaI. Finally, $p H T 43$ and $p H T 43-H A$ were transformed into B. subtilis WB800N, respectively, by electroporation as previously described ${ }^{12}$, the recombinant B. subtilis WB800N strains were named B.S. and B.S.-HA, respectively.

Analysis of fusion protein. B.S. and B.S.-HA were grown in LB medium with $5 \mu \mathrm{g} / \mathrm{ml}$ chloramphenicol, adding IPTG $(0.1 \mathrm{M})$ at growth phase $\left(\mathrm{OD}_{600}=0.5\right)$, then grown for $3 \mathrm{~h}$. The bacterial were washed three times with sterile phosphate-buffered saline (PBS) and collected. Then the B.S. and B.S.-HA was ultrasonicated. For immunodetection of the fusion proteins by western blotting ${ }^{13}$, mouse anti-HA (Abcam, USA), followed by HRP-conjugated goat anti-mouse IgG (Sigma, USA) were used. Then the blots were developed by enhanced chemiluminescence.

Isolation and culture of chicken bone marrow derived dendritic cells (BM-DCs). Chicken BM-DCs were generated as previous method ${ }^{14}$. Femurs and tibias of 4-6 week-old chickens were removed and isolated from the surrounding muscle tissue using sterile instruments. The bones were washed three times with $0.01 \mathrm{M}$ PBS, and then both ends of the bones were cut with scissors in the dish. The marrow was flushed with $0.01 \mathrm{M}$ PBS using a $10 \mathrm{ml}$ syringe with a $0.45-\mathrm{mm}$-diameter needle. Clusters within the marrow suspension were disaggregated by vigorous pipetting. After one wash in PBS, the cells were suspended in $0.01 \mathrm{M}$ PBS and loaded onto an equal volume of Histopaque-1119 (Sigma-Aldrich, UK) and centrifuged at $2500 \mathrm{rpm}$ for $25 \mathrm{~min}$. Cells at the interface were collected and washed twice with $0.01 \mathrm{M}$ PBS.

Cells obtained from femurs and tibias were cultured at a final concentration of $2 \times 10^{6} \mathrm{cells} / \mathrm{ml}$ in six-well plates in the culture medium containing RPMI-1640 (Gibco, USA), 10\% fetal bovine serum (FBS) (Wisent, CAN), $50 \mathrm{ng} / \mathrm{ml}$ recombinant chicken GM-CSF (Abcam, USA), $10 \mathrm{ng} / \mathrm{ml} \mathrm{IL-4} \mathrm{(Kingfisher,} \mathrm{USA),} 1 \mathrm{U} / \mathrm{ml}$ penicillin and $1 \mu \mathrm{g} / \mathrm{ml}$ streptomycin, for 7 days at $37^{\circ} \mathrm{C}$ and $5 \% \mathrm{CO}_{2}$. Half of the medium was replaced with fresh complete medium at day 2 and day 4 to remove non-adherent cells (such as dead cells and granulocytes). Effects of the recombinant chicken GM-CSF and IL-4 on cell differentiation were recorded by observing cell morphology, clustering and cell growth. The cell cultures were photographed during 7 days of culture with a digital camera on an inverted microscope.

Purity and uptake phenotype assay. The chicken BM-DCs were stimulated with $0.01 \mathrm{M}$ PBS, LPS $(100 \mathrm{ng} / \mathrm{ml})$, B.S. $\left(10^{7} \mathrm{cfu} /\right.$ well $)$ and B.S. $-\mathrm{HA}\left(10^{7} \mathrm{cfu} /\right.$ well $)$ at $37^{\circ} \mathrm{C}$ and $5 \% \mathrm{CO}_{2}$ at day 6. After $24 \mathrm{~h}$, cells were harvested by gentle pipetting and washed with $0.01 \mathrm{M}$ PBS. Then, the immature BM-DCs $\left(0.5 \times 10^{6} \mathrm{cells} / \mathrm{ml}\right)$ were stained with $0.05 \mathrm{mg} / \mathrm{ml}$ of PE-conjugated mouse anti-human CD11c antibody (eBioscience, USA) and incubated for $20 \mathrm{~min}$ at room temperature. The purity of BM-DCs was evaluated based on the relative levels of CD11c expression. In addition, the mature BM-DCs were stained with $0.5 \mathrm{mg} / \mathrm{ml}$ of FITC-labeled mouse anti-chicken MHC II antibody (Abcam, USA) and then incubated for $20 \mathrm{~min}$ at room temperature, following washed with $0.01 \mathrm{M}$ PBS and centrifuged at $1500 \mathrm{rpm}$ for $5 \mathrm{~min}$. DCs uptake was determined based on the relative levels of MHC II expression. After the final wash, cells were analyzed on a FACS Calibur (BD Bioscience, Cowley, UK).

Antigen uptake assay. The chicken BM-DCs $\left(0.5 \times 10^{6}\right.$ cells $\left./ \mathrm{ml}\right)$ were incubated with DyLight $488-$ B.S. or DyLight $488-B . S .-H A\left(10^{7}\right.$ or $10^{8} \mathrm{cfu} /$ well $)$ at $37^{\circ} \mathrm{C}$ for $2 \mathrm{~h}$ or $3 \mathrm{~h}$. The DCs were washed three times with PBS and then were analyzed by FACS.

Immunization Schedule. The immunization schedule was described by other researchers ${ }^{10}$. In briefly, 10-day-old Hyline chickens were randomly divided into 4 groups of 30 chickens each and immunized. Nonimmunized control (PBS) chickens received oral administration of $150 \mu \mathrm{l}$ of $0.01 \mathrm{M}$ PBS. Groups of chickens 
were immunized orally with one of the following: $10^{10} \mathrm{cfu} / \mathrm{kg} \mathrm{B.S.,} 10^{10} \mathrm{cfu} / \mathrm{kg}$ B.S.-HA, or $2 \mathrm{ml} / \mathrm{kg}$ IAIV. All chickens were immunized again one week after the first immunization. The body weights were detected from 6 chickens in each group every week.

Collection of Samples. Blood samples were taken weekly from 6 chickens in each group after the first immunization and allowed to clot overnight at room temperature before serum was collected. Serum was separated by centrifugation and stored at $-20^{\circ} \mathrm{C}$ for detection of specific IgG. The chickens were killed $1,3,5$, and 7 weeks after the first immunization. Trachea and small intestine tissue samples were taken from 6 chickens in each group, and washed with $0.5 \mathrm{ml}$ of $0.01 \mathrm{M}$ PBS repeatedly. The suspensions were centrifuged at $5,000 \times \mathrm{g}$ for $10 \mathrm{~min}$, collected, and stored at $-20^{\circ} \mathrm{C}$ for specific IgA detection. The same location of the small intestine and ileum tissues from 6 chickens after 7 week immunization were fixed with Bonn's liquid. Chickens were killed at 3 days after the second immunization, trachea and small intestine tissues and their mucosal suspensions were also collected. The mucosal suspensions were stored at $-20^{\circ} \mathrm{C}$ for detection of cytokines, and tissues stored in liquid nitrogen for TLRs expression by real-time quantitative PCR (RT-qPCR).

Hematoxylin-eosin staining assay. The same location of small intestine and ileum tissues were fixed with Bonn's liquid, embedded in paraffin and sectioned at $4 \mu \mathrm{m}$ thickness. Hematoxylin-eosin staining was applied to the paraffin sections ${ }^{15}$. Then visualized by OLYMPUS CX23. The height of small intestine villi in the same location of the small intestine and the lymph tissue area of ileum at ten different fields in each chicken were counted for the statistical analysis.

RNA isolation and quantitative RT-qPCR assay. Total RNA was extracted from trachea and small intestine tissues using a RNA extraction kit (Thermo Scientific) and subjected to reverse transcription with Prime Script RT-qPCR Kit (Takara, Dalian, CA), using an ABI 7500 instrument (Life Technologies) ${ }^{16}$. The data were reported as values normalized to a housekeeping gene ( $\beta$-actin) to account for repeated measures. Specific primers were shown in Table 1.

Enzyme-linked immunosorbent assay. Specific IgG in serum and IgA in mucosal suspensions were determined by enzyme-linked immunosorbent assay (ELISA) as previously described ${ }^{17}$. In brief, ELISA plates were coated with the purified recombinant HA protein (our laboratory saved) in carbonate buffer $(\mathrm{pH}=9.6)$ at $4{ }^{\circ} \mathrm{C}$ overnight. Plates were saturated with PBS containing $1 \%$ BSA at $37^{\circ} \mathrm{C}$ for $2 \mathrm{~h}$. The serum and mucosal suspensions of dilution degrees were added and incubated for $1 \mathrm{~h}$ at $37^{\circ} \mathrm{C}$. Subsequently, the HRP-conjugated goat anti-chicken IgG and goat anti-chicken IgA (BETHYL, CA) were added to the wells and incubated for an additional $1 \mathrm{~h}$ at $37^{\circ} \mathrm{C}$. Subsequently, a substrate solution containing o-phenylenediamine (OPD) and $\mathrm{H}_{2} \mathrm{O}_{2}$ was added, the reaction was allowed to proceed for $15 \mathrm{~min}$ at room temperature before it was terminated by the stop solution. Finally, the absorbance was measured at $450 \mathrm{~nm}$, using an automated ELISA reader (Molecular Devices, Shanghai, CA).

Hemagglutination inhibition assay. To explore whether chicken generated avian influenza virus (AIV) neutralizing antibodies, the neutralization activity of serum antibody was determined by hemagglutination inhibition (HI) assays according to the Chinese National Standard of diagnostic techniques for highly pathogenic avian influenza and the Office International des Epizooties (OIE) standards (OIE, 2005).

T-cell proliferation assays. The splenic lymphocyte were isolated from the all group chicken spleens after 7 week immunization, and then labelled with CFSE (Invitrogen, USA). Cells were cultured in a lymphocyte culture medium of $2 \times 10^{5}$ cells per well in 24 -well culture plates and stimulated by the purified recombinant HA protein (final concentration $10 \mu \mathrm{g} / \mathrm{ml}$ ) for $72 \mathrm{~h}$ at $37^{\circ} \mathrm{C}$ and $5 \% \mathrm{CO}_{2}$. Cell proliferation assays were detected by FACS. Non-stimulated cells were used as negative controls.

Statistical analysis. The results were expressed as the means \pm the standard deviations (SD). Statistical significance was determined by oneway analysis of variance (ANOVA) followed by Dunnett's t test to evaluate variations between groups; a $P$ value of $<0.05$ was considered statistically significant.

\section{Results}

The construction of recombance plasmids and the analysis of fusion protein expression. The HA fragment (1702 bp) was inserted into the pHT43 vector to generate the $p H T 43-H A$ shuttle vector (supplementary material Fig. 1A) and express HA in B. subtilis WB800N. We confirmed the presence of $p H T 43-H A$ by restriction enzyme digestion with BamHI and SmaI (supplementary material Fig. 1B). The pHT43 or $p H T 43-H A$ plasmid was transformed into $B$. subtilis $\mathrm{WB} 800 \mathrm{~N}$ by electroporation, and the HA protein was immunodetected using an HA antibody. As shown in supplementary material Fig. 1C, the lane 1 and 2 were the pHT43 plasmid group B.S., the lane 3, 4, 5, and 6 were the recombinant $p H T 43-H A$ plasmid group B.S.-HA. Immunodetection revealed a clear positive band at $63 \mathrm{KDa}$ in the recombinant $p H T 43-H A$ plasmid group B.S.-HA. The results show that the HA protein was successfully expressed in B. subtilis WB800N.

The culture of chicken bone marrow derived dendritic cells (BM-DCs) in vitro. When suspensions of chicken bone marrow cells were cultured in the presence of granulocyte-macrophage colony-stimulating factor (GM-CSF) and interleukin (IL-)-4, the immature BM-DCs aggregates had attached to a layer of adherent cells by day 4 (Fig. 1A). These aggregates grew in size and were found to be floating or loosely adherent on day 7 (Fig. 1B), following B.S., B.S.-HA, and positive lipopolysaccharide (LPS) stimulation. Many individual cells and peripheral aggregates displayed a large veiled or dendritic appearance indicating maturation (Fig. 1C). When 

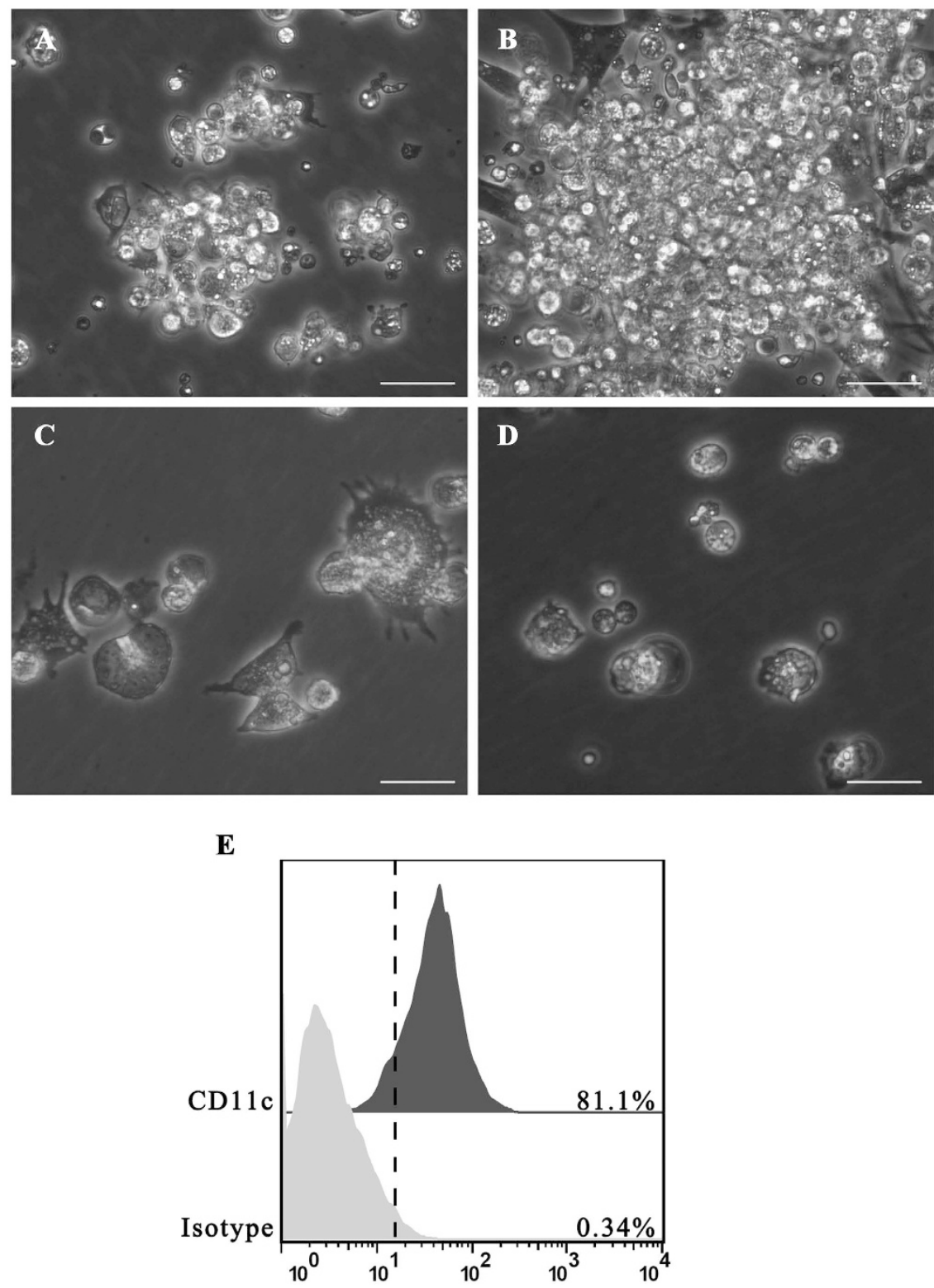

Figure 1. Morphology of chicken BM-DCs cultured with recombinant chicken GM-CSF and IL-4. (A) Light microscopy showed cell aggregates after 4 days of culture $(100 \times)$. (B) The size of these aggregates increased after $24 \mathrm{~h}$ of stimulation with B.S. or B.S.-HA $(100 \times)$. (C) Morphology of cells after 7 days of culture after stimulation with B.S. or B.S.-HA $(400 \times)$. (D) Culture of chicken bone marrow cells without chicken GM-CSF and IL-4 $(100 \times)$. Scale bars $=20 \mu \mathrm{m}$. $\left(\right.$ E) More than $81 \%$ of immature chicken BM-DCs were CD $11 c^{+}$by flow cytometry.

chicken bone marrow cells were cultured under the same conditions without GM-CSF or IL-4, no cell aggregates were observed, and only a few live cells remained in the plates by day 7 (Fig. 1D). Flow cytometry analysis of the cultured cells harvested on culture day 7 is shown in Fig. 1E. The immature DCs expressed high levels of cell surface and putative CD11c molecules.

The improvement of the BM-DCs uptake. Uptake is an important function of DCs. To explore whether B.S.-HA improves uptake by BM-DCs, different doses of DyLight 488 labelled B.S. or B.S.-HA were incubated with BM-DCs at $37^{\circ} \mathrm{C}$ for 2 or $3 \mathrm{~h}$, and the percentage of BM-DCs containing DyLight 488-B.S. or DyLight 488-B.S.-HA was detected by flow cytometry (Fig. 2A). The percentage of BM-DCs (Fig. 2B) containing DyLight 488-B.S. or DyLight 488-B.S.-HA increased $(P<0.01)$ with the dose of B.S. or B.S.-HA. The positive ratio of DCs exhibiting DyLight 488 fluorescence increased in the B.S. group, but decreased in the B.S.-HA group with the treatment time. In addition, expression of the chicken BM-DCs MHC II phenotype increased significantly after stimulated with B.S. or B.S.-HA (Fig. 3A-F) $(\mathrm{P}<0.01)$. 
A
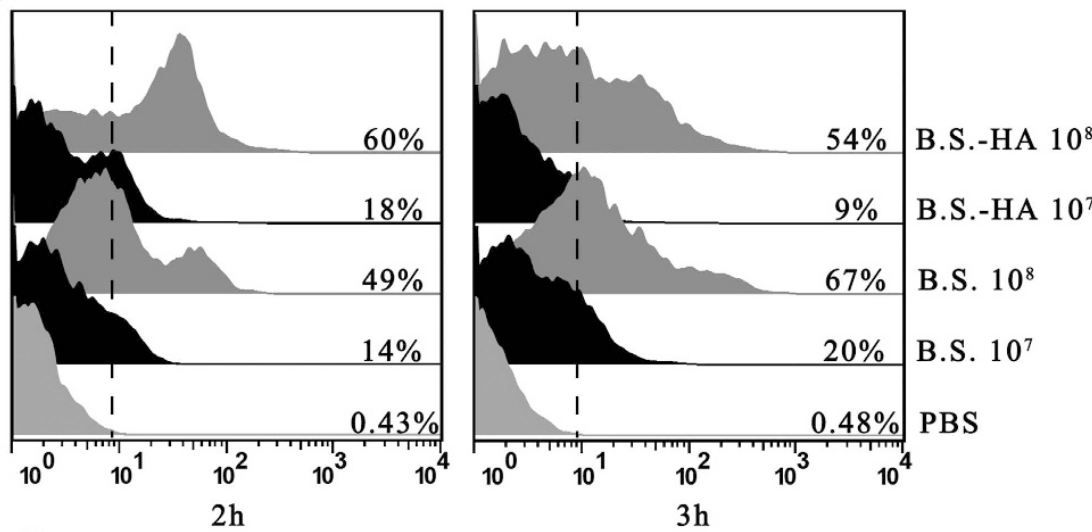

B

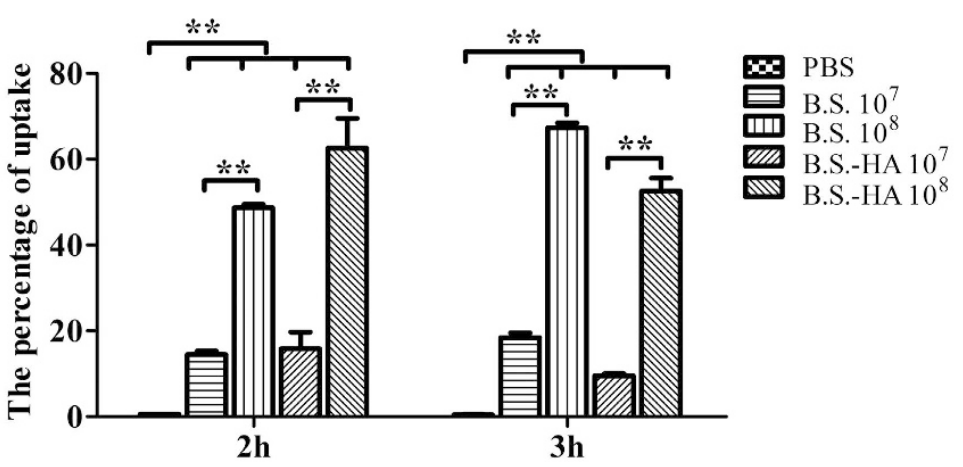

Figure 2. The analysis of B. subtilis sampled by BM-DCs. (A) The chicken BM-DCs $\left(0.5 \times 10^{6} \mathrm{cells} / \mathrm{ml}\right)$ were incubated with DyLight 488 -B.S. or DyLight $488-$ B.S. $-H A\left(10^{7}\right.$ or $10^{8} \mathrm{cfu} /$ well $)$ at $37^{\circ} \mathrm{C}$ for $2 \mathrm{~h}$ or $3 \mathrm{~h}$, and analyzed by flow cytometry. (B) The data is displayed in the histogram. Data shown are the means \pm s.d. of three samples. ${ }^{\star} P<0.05 ;{ }^{\star \star} P<0.01$. The error bars represent standard deviations.

The changes of body weight, small intestinal villi height, and lymphoid tissue area. B. subtilis has been used as a feed additive to improve the microorganismal environment in the intestine ${ }^{18}$. We weighed the chickens after they developed immunity (Fig. 4). After stimulation with B.S. or B.S.-HA, a significant increase in the weight of chickens was observed $(P<0.01$ or $P<0.05)$, compared with the other groups. Due to the important role of intestinal villi in nutrient absorption, we explored whether the intestinal villi were altered in chickens fed B.S. or B.S.-HA. As shown in Fig. 4B and D, paraffin sections from the same location of the small intestine in both groups was stained with hematoxylin-eosin, and the heights of the intestinal villi were counted 7 weeks after immunization (Fig. 4C and E). After stimulation with B.S. or B.S.-HA, a significant increase in the heights of the intestinal villi was observed $(P<0.01$ or $P<0.05)$, compared with the other groups. Indicating that immunizing chickens with B.S. or B.S.-HA may effectively enhance nutrient absorption and growth rates. Interestingly, lymphoid tissue in the ileum increased significantly in the B.S.-HA group $(P<0.01$ or $P<0.05)$, compared with the PBS group after 7 week immunization. This result indicates that B.S. $-H A$ enhances the proliferation and development of intestinal lymphoid tissue.

The levels of TLRs and cytokines. These results suggest that B.S.-HA enhances the immune response in chickens. To determine whether immunization induces a mucosal cell-mediated immune response. TLR2 and TLR4 expression was measured in the trachea and small intestine, and IL-12, IL-10, and interferon (IFN-)- $\gamma$ secretion levels in the tracheal and small intestinal suspensions were measured at 3 days after the second immunization (Fig. 5). TLR2 and TLR4 mRNA expression levels in the small intestine and trachea of chickens immunized with B.S.-HA were upregulated significantly compared with PBS and inactivated avian influenza virus (IAIV) $(P<0.05$ or $P<0.01)$, besides the TLR4 mRNA expression in the small intestine. The levels of IL-12, IL-10, and IFN- $\gamma$ secreted in the small intestine and trachea of chickens immunized with B.S.-HA and IAIV were upregulated significantly compared with those of chickens immunized with PBS $(P<0.05$ or $P<0.01)$.

The proliferation of spleen lymphocytes in vitro. To examine the proliferative response of splenic lymphocytes primed with the HA protein in vitro, splenic lymphocytes were isolated from chickens 7 weeks after immunization. After the splenic lymphocytes were stimulated by HA protein in vitro, their proliferative index was increased markedly after immunization with B.S.-HA $(P<0.01)$ (Fig. 6).

The changes of AIV-specific immune responses. Specific IgA antibody levels were measured 1, 3, 5, and 7 weeks after the first immunization. High levels of mucosal specific IgA antibody were observed in the tracheal 

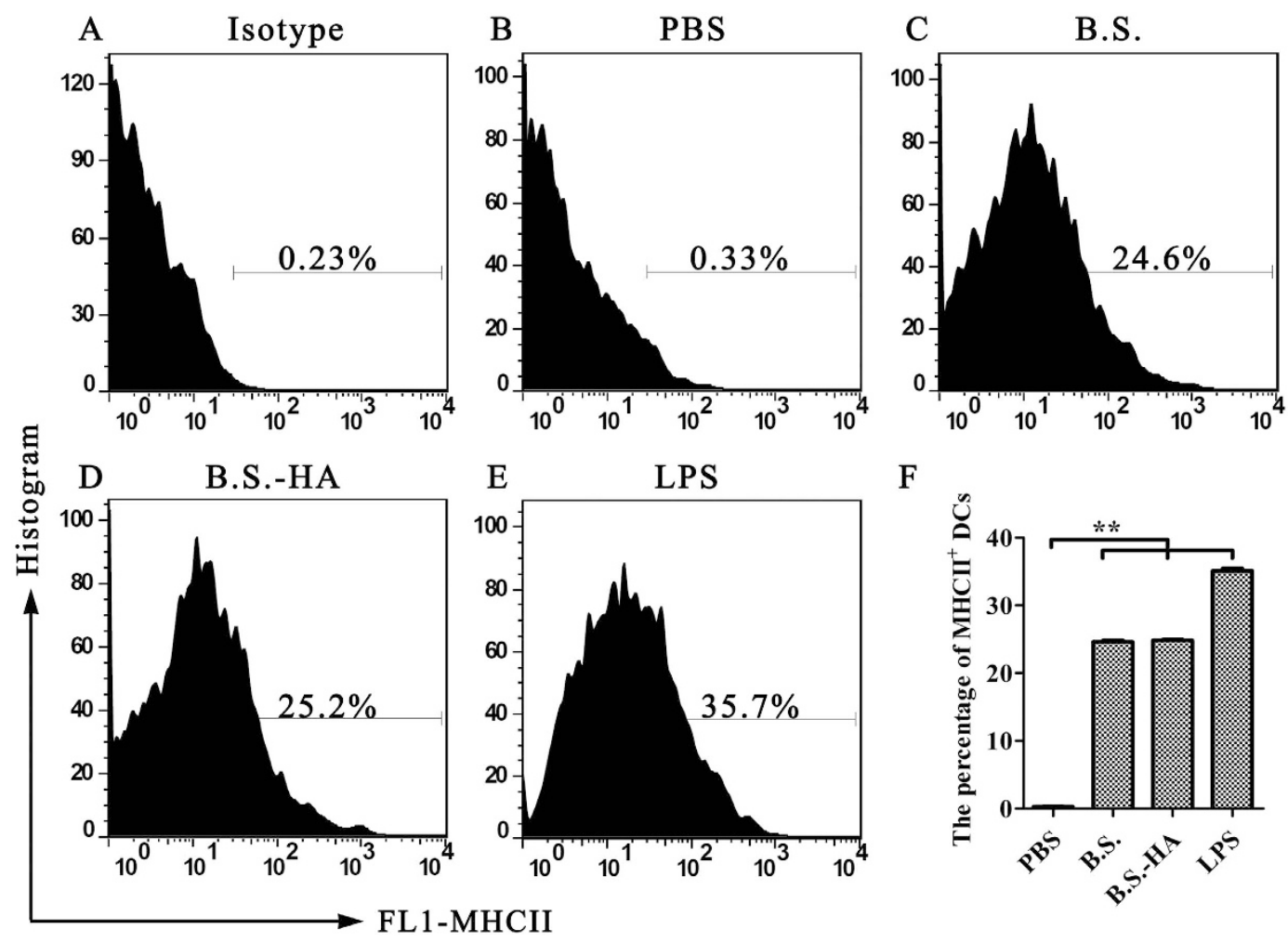

F

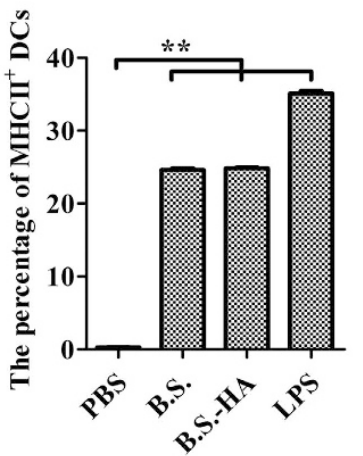

Figure 3. The analysis of MHC II phenotype expression in BM-DCs. (A-E) The chicken BM-DCs were stimulated with PBS, LPS $(100 \mathrm{ng} / \mathrm{ml})$, B.S. ( $10^{7} \mathrm{cfu} /$ well $)$ and B.S. $-H A\left(10^{7} \mathrm{cfu} /\right.$ well $)$ for $24 \mathrm{~h}$, stained with $0.5 \mathrm{mg} / \mathrm{ml}$ of FITC-labeled mouse anti-chicken MHCII antibody, and analyzed by flow cytometry. (F) The data is displayed in the histogram. Data shown are the means \pm s.d. of three samples. ${ }^{\star} P<0.05 ;{ }^{*} P<0.01$. The error bars represent standard deviations.

suspensions and increased significantly 3 and 5 weeks after oral immunization with B.S.-HA $(\mathrm{P}<0.01$; Fig. 7A) compared with immunized with PBS or B.S. The level of mucosal specific IgA antibody was also increased in the group immunized with IAIV.

Specific immunity can be effectively promoted by cellular and innate immunities. Serum IgG antibody levels were detected 1-7 weeks after the first immunization. Serum antibody levels increased ( $<0.01$; Fig. $7 \mathrm{~B})$, peaking at 3-5 weeks after immunization with B.S.-HA compared with immunization with PBS or B.S. In addition, the IAIV induced higher levels of IgG antibodies at weeks 3 and 4.

The serum antibody titer was detected by the HI assay (Fig. 7C). Serum titers from group that immunized with B.S.-HA increased at 3 weeks and peaked at 5 weeks after the first immunization. In addition, IAIV induced a higher titer 4 weeks after the first immunization. These results show that oral administration of B.S.-HA effectively induced mucosal immunity, a systemic immune response, and resistance to the infection by AIV to a certain extent.

\section{Discussion}

B. subtilis is a well-characterized spore-forming bacterium widely used to express heterologous proteins and deliver antigens ${ }^{8,19-21}$. B. subtilis is a probiotic microorganism in broilers and laying hens ${ }^{22-24}$. Previous studies have indicated that dietary supplementation of B. subtilis exerts a beneficial effect on intestinal microbiota and gut morphology thereby enhancing growth performance and improving the feed conversion ratio in animals ${ }^{18}$. These effects of $B$. subtilis are probably due to its ability to produce amylase, protease, lipase and amino acids ${ }^{25}$, which could increase the efficiency of digestion and absorption of nutrients. Researchers generally agree that the improved growth performance observed in response to probiotics is associated with healthy modulation of the gut community composition ${ }^{26}$. In addition, B. subtilis has been used as an adjuvant to stimulate the immune response ${ }^{27}$.

DCs are the most potent professional antigen-presenting cells and play a crucial role in linking of innate and adaptive immunities ${ }^{28}$. B. subtilis recruits more DCs, migrates to mesenteric lymph nodes, and induces an immune response. Our previous experiments showed that $B$. subtilis increases the MHC II phenotype in pig $\mathrm{BM}-\mathrm{DC}$ in vitro and stimulates the uptake ability of $\mathrm{DCs}^{29}$. In this study, recombinant $B$. subtilis was sampled by BM-DCs in vitro, and the percentage of BM-DCs containing B. subtilis increased with the quantity of bacteria used. Interestingly, the quantity of the recombinant bacteria in DCs decreased as the incubation time extended. It reported that recombinant HA proteins from $\mathrm{H} 5 \mathrm{~N} 1$ influenza viruses are capable of activating mouse DCs activation and suppressing endocytosis ${ }^{30}$. After the B.S.-HA sampled by chicken BM-DCs a period of time, the exposed HA protein inhibited the endocytosis of chicken BM-DCs. 


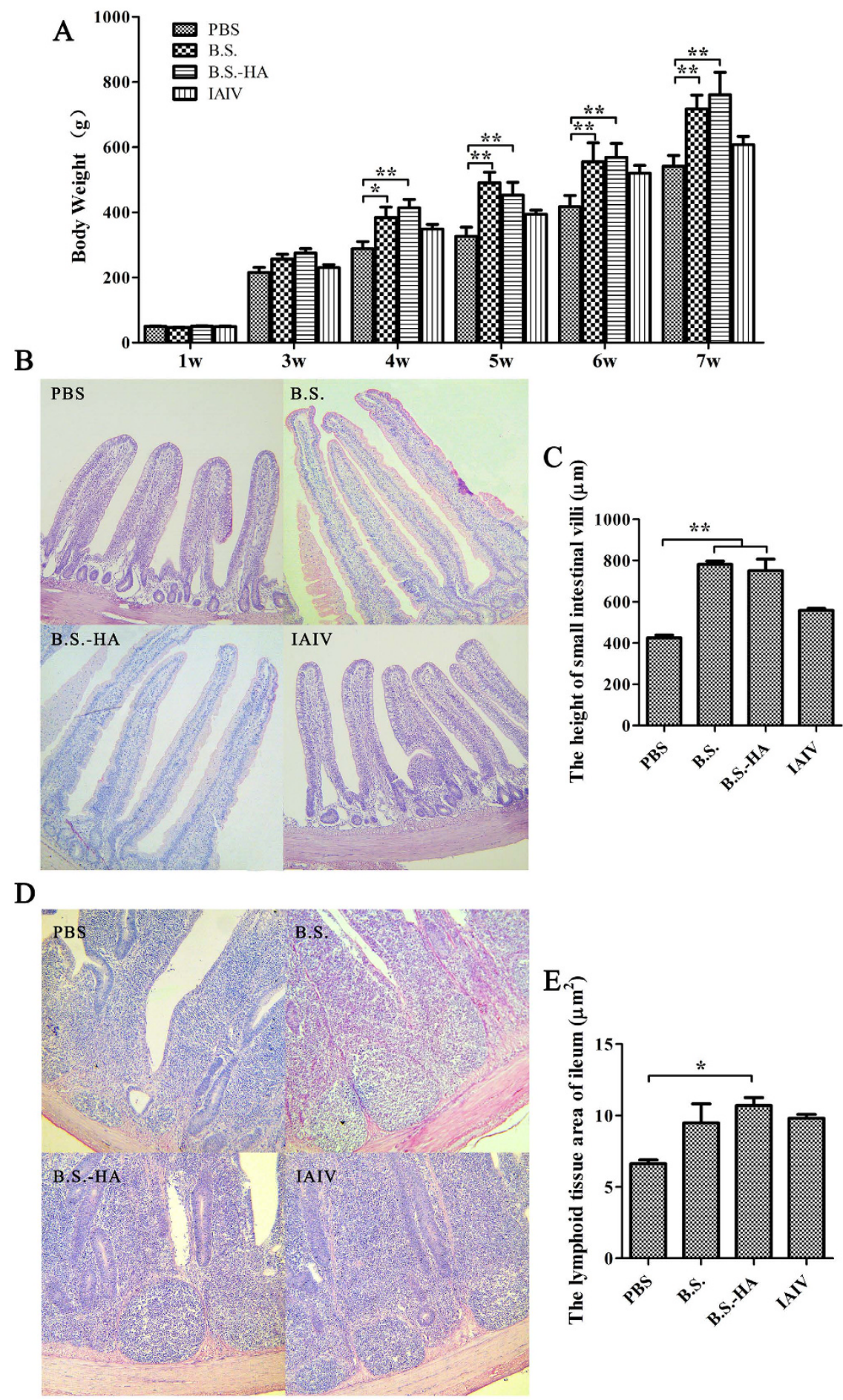

Figure 4. Changes of body weight, small intestinal villi height and lymphoid tissue area. (A) The body weights were counted in each group every week. (B) Hematoxylin-eosin stained on the same part of the small intestine and detected the intestinal villi height after immunized 7 weeks $(100 \times)$. (C) The data is displayed in the histogram. (D) Hematoxylin-eosin stained on ileum and detected the lymphoid tissue area after immunized 7 weeks $(400 \times)$. (E) The data is displayed in the histogram. Data shown are the means \pm s.d. of six samples. ${ }^{\star} P<0.05 ;{ }^{*} P<0.01$. The error bars represent standard deviations.

TLRs are required to activate innate and adaptive immune responses ${ }^{31,32}$. B. subtilis is recognized by TLR2 or TLR4 and augments mucosal and systemic responses to intranasal antigens in mice ${ }^{33,34}$. B. subtilis could activate the nuclear factor kappa B pathway by binding to TLRs and induced production of primary T helper type 1 (Th1) cytokines such as IL-1, IFN- $\gamma$, and IL-12 ${ }^{35}$. Our results show that B.S. $-H A$ upregulate secretion of IFN- $\gamma$ and IL-12 in the chicken small intestine and trachea. These cytokines further activated T cells, consequently enhanced 
$\mathbf{A}$

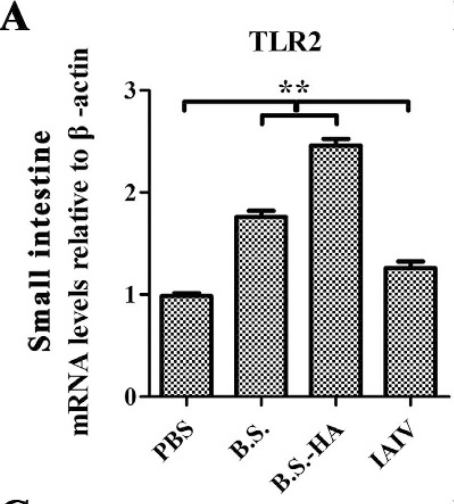

C

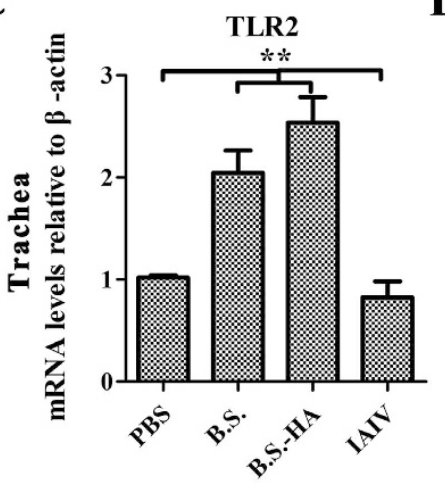

B

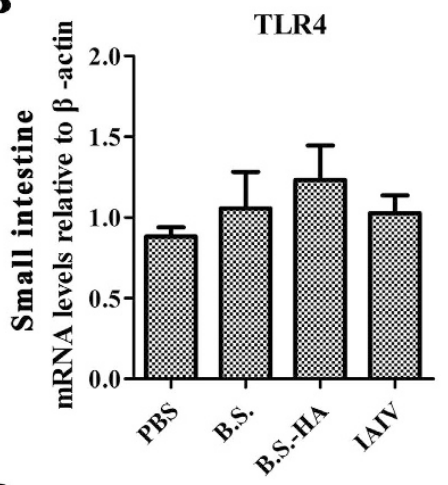

D

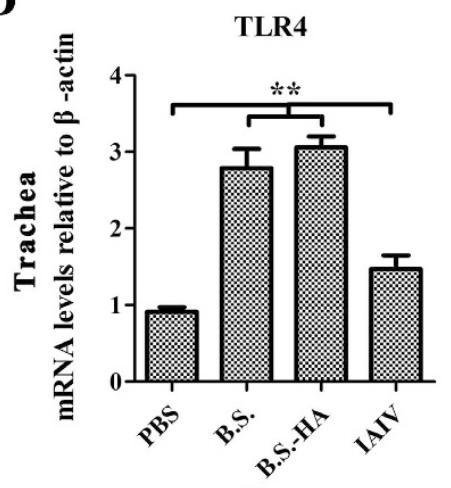

$\mathbf{E}$

$$
\text { IL-12 }
$$

F

IL-10

$\mathbf{G}$

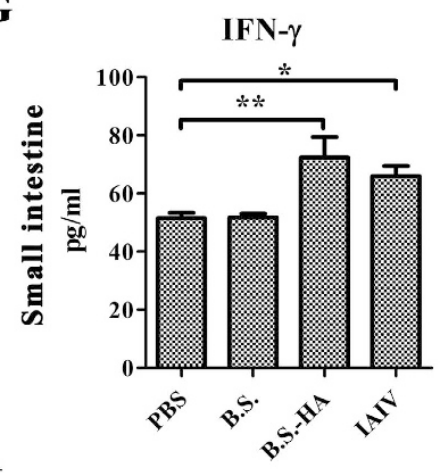

$\mathbf{H}$

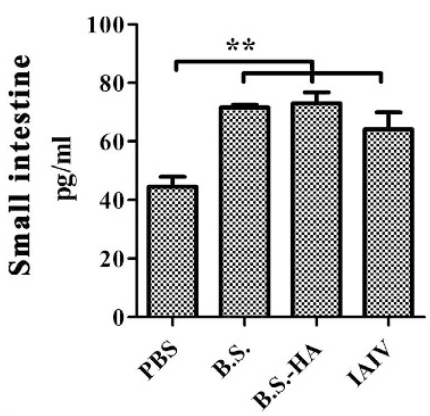

IL-12

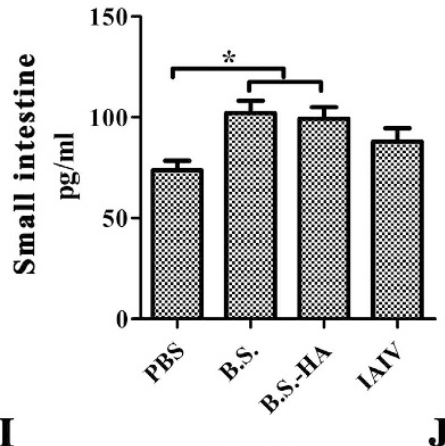

IL-10

IFN- $\gamma$
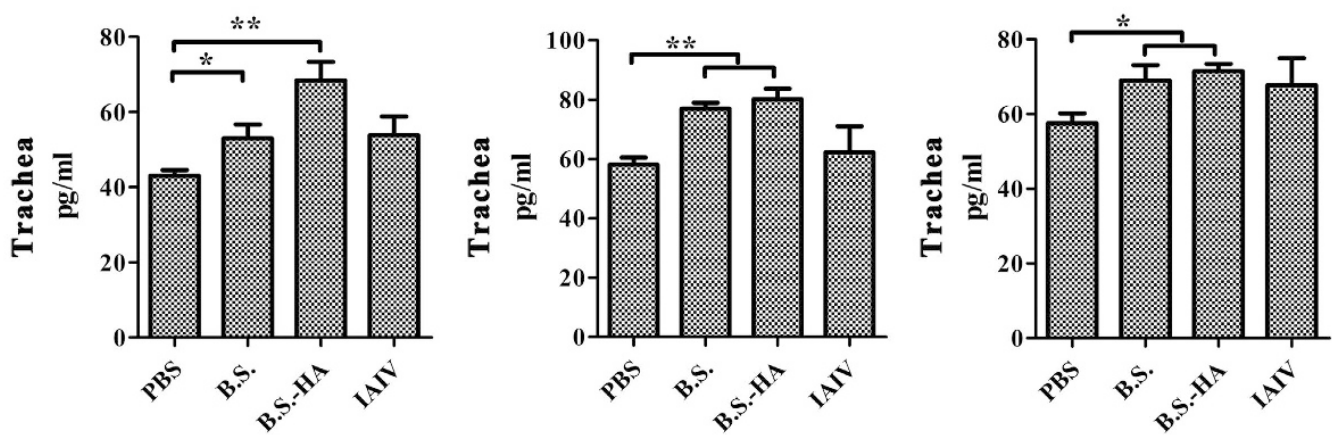

Figure 5. The expression levels of Toll-like receptors (TLRs) and cytokines. TLR2 and TLR4 mRNA expression in the small intestine $(\mathbf{A}, \mathbf{B})$ and trachea $(\mathbf{C}, \mathbf{D})$ were measured at day 3 after the second immunization. IL-12, IL-10, and IFN- $\gamma$ cytokines expression levels in the small intestine (E-G) and trachea $(\mathbf{H}-\mathbf{J})$ suspensions were measured at day 3 after the second immunization. Data shown are the means \pm s.d. of six samples. ${ }^{\star} P<0.05 ;{ }^{* \star} P<0.01$. The error bars represent standard deviations.

immune response. The IL-10 as Th2-related cytokine, which could be induced by B.S.-HA in chickens small intestine and trachea, can promote the maturation and activation of $B$ cells, further increase the production of antigen specific IgA and IgG antibodies ${ }^{36}$. 
A

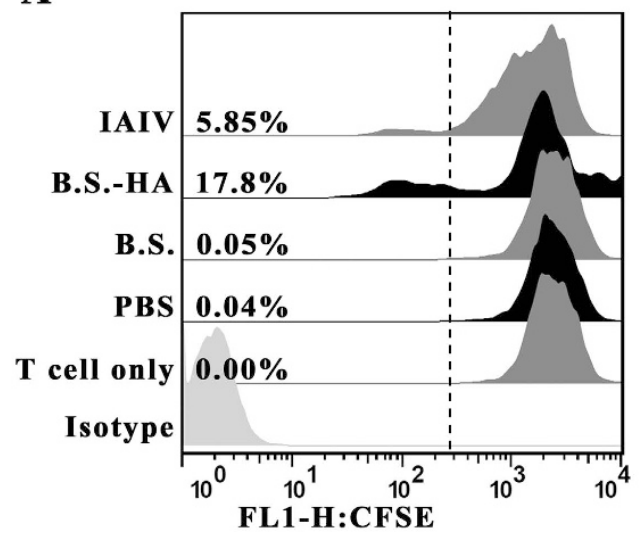

B

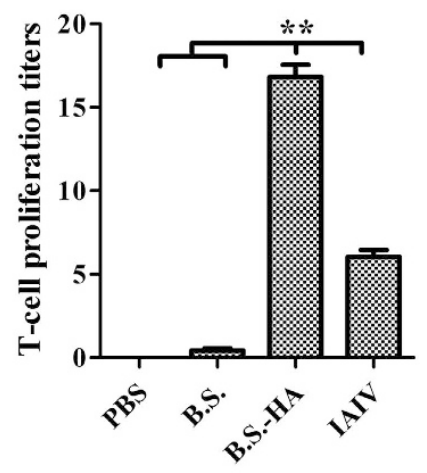

Figure 6. The proliferation of spleen lymphocytes in vitro. (A) HA-specific splenic lymphocyte proliferative reaction. Cells were cultured in a lymphocyte culture medium of $2 \times 10^{5}$ cells per well in 24 -well culture plates and stimulated by the purified recombinant HA protein (final concentration $10 \mu \mathrm{g} / \mathrm{ml}$ ) for $72 \mathrm{~h}$. Cell proliferation assays were detected by flow cytometry. Non-stimulated cells were used as negative controls; (B) The data is displayed in the histogram. Data shown are the means \pm s.d. of three samples. ${ }^{\star} P<0.05 ;{ }^{* *} P<0.01$. The error bars represent standard deviations.

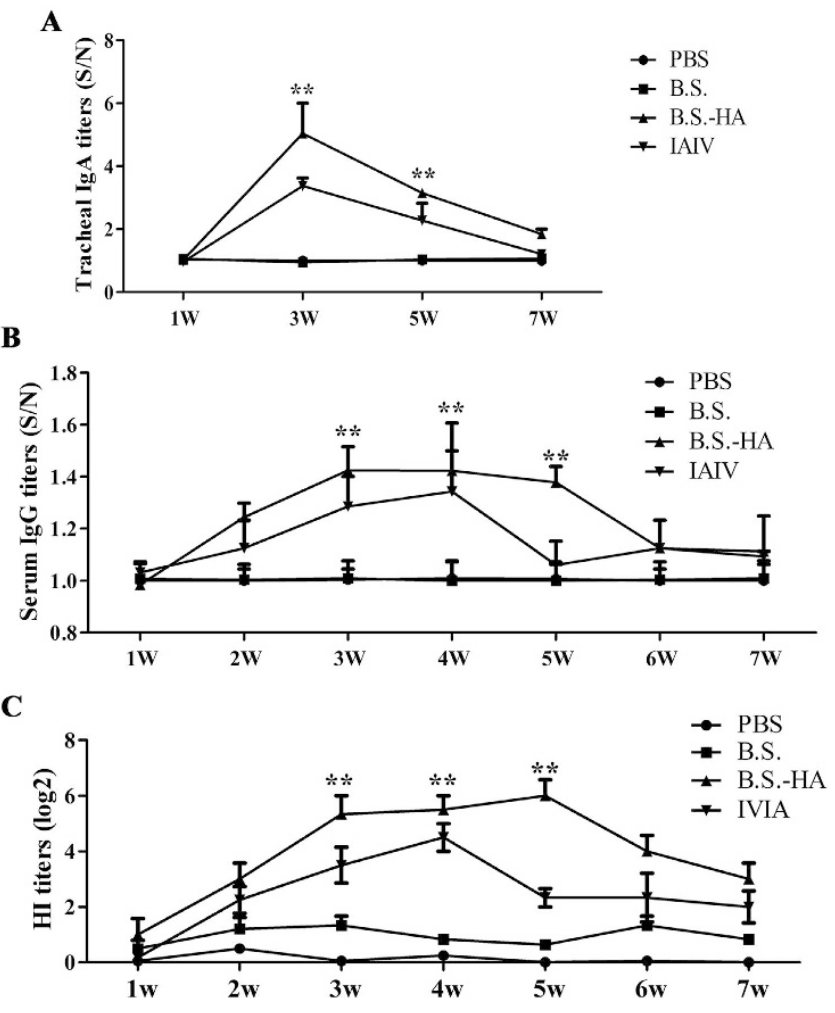

Figure 7. B.S.-HA enhanced mucosal and systemic immune responses after oral immunization. (A) The trachea suspensions after 1, 3, 5, and 7 week immunization were analyzed specific IgA (S/N) by indirect ELISA. (B) Serum after 1-7 week immunization were analyzed specific IgG $(\mathrm{S} / \mathrm{N})$ by indirect ELISA. (C) HI antibodies in serum were analyzed by hemagglutination inhibition (HI) assays. Data shown are the means \pm s.d. of six samples. ${ }^{\star} P<0.05 ;{ }^{\star *} P<0.01$. The error bars represent standard deviations.

According to the "common mucosal immune system" theory, antigen-sensitized precursor B and T lymphocytes generated at one mucosal site (such as the gut) can be detected at anatomically remote and functionally distinct compartments (such as the respiratory mucosa) ${ }^{37}$. In addition, avian influenza H5N1 has been detected in the nasal cavity mucosa, lungs, cloaca, and serum of aerosol-infected chickens ${ }^{38}$. Secretory IgA antibodies are the major contributors to humoral mucosal immunity against an influenza viral infection ${ }^{39}$. Our results show that oral administration of B.S.-HA increased the specific IgA antibody level in tracheal suspensions. The mucosal 
vaccine induced mucosal and systemic immune responses. Systemic immunity plays an important role in preventing the $\mathrm{H} 5 \mathrm{~N} 1$ virus. In this study, serum specific IgG and $\mathrm{HI}$ antibody titers were boosted by oral immunization of B.S.-HA, indicating that the recombinant B. subtilis effectively stimulated the systemic immune response.

\section{Conclusion}

This study indicated that the recombinant B.S.-HA upregulated the MHC II phenotype expression in BM-DCs to sample B.S.-HA, and induced immune responses. Immunized chickens with B.S.-HA significantly elevated specific IgA titers in trachea, IgG and HI antibody titers in serum.

\section{References}

1. Ungchusak, K. et al. Probable person-to-person transmission of avian influenza A (H5N1). The New England journal of medicine 352, 333-340, doi: 10.1056/NEJMoa044021 (2005).

2. Niu, Z. Y., Liu, F. Z., Yan, Q. L. \& Li, W. C. Effects of different levels of vitamin E on growth performance and immune responses of broilers under heat stress. Poultry science 88, 2101-2107, doi: 10.3382/ps.2009-00220 (2009).

3. Treanor, J. J. et al. Safety and immunogenicity of a recombinant hemagglutinin vaccine for H5 influenza in humans. Vaccine 19, 1732-1737 (2001).

4. Chen, Z. et al. Protection and antibody responses in different strains of mouse immunized with plasmid DNAs encoding influenza virus haemagglutinin, neuraminidase and nucleoprotein. The Journal of general virology 80 ( Pt 10), 2559-2564, doi: 10.1099/00221317-80-10-2559 (1999).

5. Toro, H. et al. Protective avian influenza in ovo vaccination with non-replicating human adenovirus vector. Vaccine 25, 2886-2891, doi: 10.1016/j.vaccine.2006.09.047 (2007).

6. Gu, J. et al. H5N1 infection of the respiratory tract and beyond: a molecular pathology study. Lancet 370, 1137-1145, doi: 10.1016/ S0140-6736(07)61515-3 (2007).

7. Driks, A. Bacillus subtilis spore coat. Microbiol Mol Biol Rev 63, 1-20 (1999).

8. Ferreira, L. C., Ferreira, R. C. \& Schumann, W. Bacillus subtilis as a tool for vaccine development: from antigen factories to delivery vectors. An Acad Bras Cienc 77, 113-124, doi: /S0001-37652005000100009 (2005).

9. Hoess, R. H. Bacteriophage lambda as a vehicle for peptide and protein display. Curr Pharm Biotechnol 3, 23-28 (2002).

10. Liang, J. et al. Comparison of 3 kinds of Toll-like receptor ligands for inactivated avian H5N1 influenza virus intranasal immunization in chicken. Poultry science 92, 2651-2660, doi: 10.3382/ps.2013-03193 (2013).

11. Amuguni, H. \& Tzipori, S. Bacillus subtilis: a temperature resistant and needle free delivery system of immunogens. Human vaccines \& immunotherapeutics 8, 979-986, doi: 10.4161/hv.20694 (2012).

12. Narita, J. et al. Display of alpha-amylase on the surface of Lactobacillus casei cells by use of the PgsA anchor protein, and production of lactic acid from starch. Applied and environmental microbiology 72, 269-275, doi: 10.1128/AEM.72.1.269-275.2006 (2006).

13. Wang, Z., Yu, Q., Gao, J. \& Yang, Q. Mucosal and systemic immune responses induced by recombinant Lactobacillus spp. expressing the hemagglutinin of the avian influenza virus H5N1. Clin Vaccine Immunol 19, 174-179, doi: 10.1128/CVI.05618-11 (2012).

14. Liang, J. et al. The stimulatory effect of TLRs ligands on maturation of chicken bone marrow-derived dendritic cells. Veterinary immunology and immunopathology 155, 205-210, doi: 10.1016/j.vetimm.2013.06.014 (2013).

15. Kang, H., Yan, M., Yu, Q. \& Yang, Q. Characteristics of nasal-associated lymphoid tissue (NALT) and nasal absorption capacity in chicken. PLoS One 8, e84097, doi: 10.1371/journal.pone.0084097 (2013).

16. Wang, Z., Yu, Q., Fu, J., Liang, J. \& Yang, Q. Immune responses of chickens inoculated with recombinant Lactobacillus expressing the haemagglutinin of the avian influenza virus. J Appl Microbiol 115, 1269-1277, doi: 10.1111/jam.12325 (2013).

17. Mou, C., Zhu, L., Xing, X. \& Q. Y. Expression of major antigenic sites A and D in S gene of Transmissible gastroenteritis virus of swine (TGEV) in Escherichia coli and development of indirect ELISA for detection of the antibody against TGEV. Chinese veterinary science 45, 356-360 (2015).

18. Lei, X. et al. Effect of Bacillus amyloliquefaciens-based Direct-fed Microbial on Performance, Nutrient Utilization, Intestinal Morphology and Cecal Microflora in Broiler Chickens. Asian-Australasian journal of animal sciences 28, 239-246, doi: doi: 10.5713/ ajas.14.0330 (2015)

19. Gat, O. et al. Use of a promoter trap system in Bacillus anthracis and Bacillus subtilis for the development of recombinant protective antigen-based vaccines. Infect Immun 71, 801-813 (2003).

20. Olmos-Soto, J. \& Contreras-Flores, R. Genetic system constructed to overproduce and secrete proinsulin in Bacillus subtilis. Appl Microbiol Biotechnol 62, 369-373, doi: 10.1007/s00253-003-1289-4 (2003).

21. Mauriello, E. M. et al. Display of heterologous antigens on the Bacillus subtilis spore coat using CotC as a fusion partner. Vaccine 22, 1177-1187, doi: 10.1016/j.vaccine.2003.09.031 (2004).

22. Chen, K. L. et al. Effects of Bacillus subtilis var. natto and Saccharomyces cerevisiae mixed fermented feed on the enhanced growth performance of broilers. Poultry science 88, 309-315, doi: 10.3382/ps.2008-00224 (2009).

23. Kurtoglu, V. et al. Effect of probiotic supplementation on laying hen diets on yield performance and serum and egg yolk cholesterol. Food additives and contaminants 21, 817-823, doi: 10.1080/02652030310001639530 (2004).

24. Liu, X. et al. Growth Performance and Meat Quality of Broiler Chickens Supplemented with Bacillus licheniformis in Drinking Water. Asian-Australasian journal of animal sciences 25, 682-689, doi: 10.5713/ajas.2011.11334 (2012).

25. Santoso, U., Tanaka, K. \& Ohtani, S. Effect of dried Bacillus subtilis culture on growth, body composition and hepatic lipogenic enzyme activity in female broiler chicks. The British journal of nutrition 74, 523-529 (1995).

26. Pedroso, A. A. et al. Intestinal bacterial community and growth performance of chickens fed diets containing antibiotics. Poultry science 85, 747-752 (2006).

27. Li, Y., Li, P., Wang, X., Yu, Q. \& Yang, Q. Co-administration of attenuated Mycoplasma hyopneumoniae 168 strain with bacterial DNA enhances the local and systemic immune response after intranasal vaccination in pigs. Vaccine 30, 2153-2158, doi: 10.1016/j. vaccine.2012.01.028 (2012)

28. Liu, W. C. et al. Influenza Hemagglutinin Glycoproteins with Different N-glycan Patterns Activate Dendritic Cells in Vitro. Journal of virology, doi: 10.1128/JVI.00452-16 (2016).

29. Mou, C., Zhu, L., Xing, X., Lin, J. \& Yang, Q. Immune responses induced by recombinant Bacillus subtilis expressing the spike protein of transmissible gastroenteritis virus in pigs. Antiviral research 131, 74-84, doi: 10.1016/j.antiviral.2016.02.003 (2016).

30. Liu, W. C., Lin, S. C., Yu, Y. L., Chu, C. L. \& Wu, S. C. Dendritic cell activation by recombinant hemagglutinin proteins of H1N1 and H5N1 influenza A viruses. Journal of virology 84, 12011-12017, doi: 10.1128/JVI.01316-10 (2010).

31. Lahiri, A., Das, P. \& Chakravortty, D. Engagement of TLR signaling as adjuvant: towards smarter vaccine and beyond. Vaccine 26, 6777-6783, doi: 10.1016/j.vaccine.2008.09.045 (2008).

32. Kumar, H., Kawai, T. \& Akira, S. Toll-like receptors and innate immunity. Biochemical and biophysical research communications 388, 621-625, doi: 10.1016/j.bbrc.2009.08.062 (2009).

33. Huang, J. M. et al. Mucosal delivery of antigens using adsorption to bacterial spores. Vaccine 28, 1021-1030, doi: 10.1016/j. vaccine.2009.10.127 (2010) 
34. Barnes, A. G., Cerovic, V., Hobson, P. S. \& Klavinskis, L. S. Bacillus subtilis spores: a novel microparticle adjuvant which can instruct a balanced Th1 and Th2 immune response to specific antigen. European journal of immunology 37, 1538-1547, doi: 10.1002/ eji.200636875 (2007)

35. Schneider-Ohrum, K. et al. Adjuvants that stimulate TLR3 or NLPR3 pathways enhance the efficiency of influenza virus-like particle vaccines in aged mice. Vaccine 29, 9081-9092, doi: 10.1016/j.vaccine.2011.09.051 (2011).

36. Burdin, N. et al. Endogenous IL-6 and IL-10 contribute to the differentiation of CD40-activated human B lymphocytes. Journal of immunology 154, 2533-2544 (1995).

37. Buchanan, R. M., Mertins, S. \& Wilson, H. L. Oral antigen exposure in extreme early life in lambs influences the magnitude of the immune response which can be generated in later life. BMC veterinary research 9, 160, doi: 10.1186/1746-6148-9-160 (2013).

38. Sergeev, A. A. et al. Infection of chickens caused by avian influenza virus A/H5N1 delivered by aerosol and other routes. Transboundary and emerging diseases 60, 159-165, doi: 10.1111/j.1865-1682.2012.01329.x (2013).

39. Suzuki, T. et al. Relationship of the quaternary structure of human secretory IgA to neutralization of influenza virus. Proceedings of the National Academy of Sciences of the United States of America 112, 7809-7814, doi: 10.1073/pnas.1503885112 (2015).

\section{Acknowledgements}

This work was supported by National Natural Science Foundation of China (31172302) and Priority Academic Program Development of Jiangsu Higher Education Institutions (PAPD).

\section{Author Contributions}

Chunxiao Mou design and performed all the experiments, analyzed the data and writed the manuscript; Liqi Zhu coordinated the study, participated in writing the manuscript; Jingjing Yang participated in BM-DCs isolation and culture; Wenwen Xu and Xiaoying Cheng participated in animal immunity experiments; Qian Yang supervised the experiment and participated in the experiment designs; All the authors read and approved the final manuscript.

\section{Additional Information}

Supplementary information accompanies this paper at http://www.nature.com/srep

Competing financial interests: The authors declare no competing financial interests.

How to cite this article: Mou, C. et al. Immune Responses Induced by Recombinant Bacillus Subtilis Expressing the Hemagglutinin Protein of H5N1 in chickens. Sci. Rep. 6, 38403; doi: 10.1038/srep38403 (2016).

Publisher's note: Springer Nature remains neutral with regard to jurisdictional claims in published maps and institutional affiliations.

(c) (i) This work is licensed under a Creative Commons Attribution 4.0 International License. The images or other third party material in this article are included in the article's Creative Commons license, unless indicated otherwise in the credit line; if the material is not included under the Creative Commons license, users will need to obtain permission from the license holder to reproduce the material. To view a copy of this license, visit http://creativecommons.org/licenses/by/4.0/

(c) The Author(s) 2016 